3. A. A. Albert, Modern higher algebra, The University of Chicago Science Series, 1937 , p. 26.

4. V. J. Varineau, An extension of the theory of matrices with elements in a principal ideal ring, This paper was submitted as his doctoral dissertation at the University of Wisconsin, July 1940.

5. J. H. Bell, Left associates of monic matrices with an application to unilateral matrix equations, Amer. J. Math. vol. 71 (1949) pp. 249-256, see p. 254.

Michigan State College

\title{
CANONICAL POSITIVE DEFINITE MATRICES UNDER INTERNAL LINEAR TRANSFORMATIONS
}

\section{BERNARD VINOGRADE}

Let $P$ be a real positive definite $n$-rowed square matrix, $n=p+q$ $+r, p \leqq q \leqq r, S=\operatorname{diag}\left(S_{1}, S_{2}, S_{3}\right)$, where $S_{1}$ is $p$-rowed, $S_{2}$ is $q$-rowed, and $S_{3}$ is $r$-rowed, all nonsingular. $S$ is called an internal linear transformation when it transforms $P$ according to the rule $P \rightarrow S P S^{\prime}$. All coefficients are real numbers. The problem is to find a canonical form for $P$, under internal linear transformations, depending on characteristic value systems associated with $P$. The partition of $n$ into three parts is for convenience only.

1. Semi-canonical reduction. Partition $P$ according to the partition of $n$, and call the square diagonal blocks $P_{1}, P_{2}$, and $P_{3}$, with $p, q$, and $r$ rows respectively. Choose $S_{1}=Q_{1}^{-1}, S_{2}=Q_{2}^{-1}, S_{3}=Q_{3}^{-1}$ from the factorizations $P_{1}=Q_{1} Q_{1}^{\prime}, P_{2}=Q_{2} Q_{2}^{\prime}, P_{3}=Q_{3} Q_{3}^{\prime}$. Then

$$
S P S^{\prime}=Q=\left(\begin{array}{ccc}
I_{p} & A & B \\
A^{\prime} & I_{q} & C \\
B^{\prime} & C^{\prime} & I_{r}
\end{array}\right),
$$

where $I_{i}$ is the $i$-rowed identity matrix. Any further transformations will be chosen so as to preserve the diagonal blocks $I_{p}, I_{q}, I_{r}$. Hence we shall have $S_{i} S_{i}^{\prime}=I_{i}, i=1,2,3$.

2. Non-special cases. Let $X=\left(I_{p}, A, B\right), \quad Y=\left(A^{\prime}, I_{q}, C\right), Z$ $=\left(B^{\prime}, C^{\prime}, I_{r}\right)$, so $X X^{\prime}, Y Y^{\prime}$, and $Z Z^{\prime}$ are all nonsingular and symmetric. Let their characteristic roots be the ordered diagonal elements of $D_{1}=\operatorname{diag}\left(\lambda_{1}, \cdots, \lambda_{p}\right), D_{2}=\operatorname{diag}\left(\mu_{1}, \cdots, \mu_{q}\right)$, and $D_{3}$

Received by the editors December 27, 1948. 
$=\operatorname{diag}\left(\nu_{1}, \cdots, \nu_{r}\right)$. If the $\lambda_{i}$ 's are distinct, then an orthogonal matrix $K_{1}$ such that $K_{1} X X^{\prime} K_{1}^{\prime}=D_{1}$ is unique within a left factor $\operatorname{diag}( \pm 1, \cdots, \pm 1)$. If also the $\mu_{i}$ 's and the $\nu_{i}$ 's are distinct respectively, then $S=\operatorname{diag}\left(K_{1}, K_{2}, K_{3}\right)$ gives $S Q S^{\prime}$ in a canonical form with rows and corresponding columns orthogonal in groups of $p, q$, and $r$, and coefficients unique within internal permutations and sign changes.

3. Special cases. After carrying out the reduction of $\$ 2$, let diag $\left(K_{1}, K_{2}, K_{3}\right)$ stand for any further orthogonal internal transformation. Suppose there are $a$ distinct $\lambda_{i}$ 's and $a<p$. For convenience of discussion, assume there are $a_{1} \lambda_{1}$ 's in the first $a_{1}$ places in $D_{1}$, $\lambda_{1} \neq 1$ and $a_{1}>1$. Then in $K_{1}$ there is a free orthogonal $a_{1}$-rowed block. Generally, $K_{1}=\operatorname{diag}\left(K_{11}, \cdots, K_{1 a}\right)$, each $K_{1 i}$ orthogonal. It follows from $(A, B)(A, B)^{\prime}=\operatorname{diag}\left(\lambda_{1}-1, \cdots, \lambda_{p}-1\right)$ that if $\left(A_{a_{1}}, B_{a_{1}}\right)$ are the first $a_{1}$ rows of $(A, B)$ then $\left(A_{a_{1}}, B_{a_{1}}\right)\left(A_{a_{1}}, B_{a_{1}}\right)^{\prime}=\left(\lambda_{1}-1\right) I_{a_{1}}$. Impose on $K_{11}$ the condition $K_{11} A_{a_{1}} A_{a_{1}} K_{11}^{\prime}=\operatorname{diag}\left(h_{1}, \cdots, h_{a_{1}}\right)$. If the $h_{i}$ 's are not distinct, suppose $h_{1}=\cdots=h_{\alpha_{1}} \neq 0$. Let $L_{1}$ be the corresponding $\alpha_{1} \times \alpha_{1}$ free orthogonal block in $K_{11}$, and $\left(A_{\alpha_{1}}, B_{\alpha_{1}}\right)$ the corresponding $\alpha_{1}$ rows, then $A_{\alpha_{1}}$ is of rank $\alpha_{1}$ and contains an $\alpha_{1} \times \alpha_{1}$ nonsingular minor array $G$. After an internal permutation suppose $A_{\alpha_{1}}=(G, H)$, then the free $L_{1}$ may be absorbed by requiring $L_{1} G$ to be the unique triangular factor (say with positive diagonal coefficients) of $G^{\prime} G$. If $h_{1}=0$ then the process starts with $B_{\alpha_{1}}$, and if the partition is into more than three parts, $B_{\alpha_{1}}$ may be orthogonalized first. If $\lambda_{1}=1$, then clearly $\left(A_{a_{1}}, B_{a_{1}}\right)=0$. None of these transformations affect the orthogonality conditions imposed by the reduction of $\$ 2 . Y$ and $Z$ are reduced similarly with the freedom in $K_{2}$ and $K_{3}$, if any.

4. The case $n=p+q$. In this case a more detailed description is possible and is derived here as a consequence of the following lemma. It has also been displayed by Hotelling as a by-product of a statistical investigation. ${ }^{1}$

Lemma. If two real matrices $A$ and $B$ are each a-rowed and $b$ columned, then $A A^{\prime}=B B^{\prime}$ if and only if $A=B U$ where $U$ is orthogonal.

Proof. $A A^{\prime}$ is an $a$-rowed symmetric matrix, hence there exists an orthogonal $V$ such that $V A A^{\prime} V^{\prime}=V B B^{\prime} V^{\prime}=\operatorname{diag}\left(d_{1}, \cdots, d_{r}\right.$, $0, \cdots, 0)$, where $r \leqq a$ and each $d_{i}$ is positive. Let $D^{-1}=\operatorname{diag}\left(1 /\left(d_{1}\right)^{1 / 2}\right.$ $\left.\cdots, 1 /\left(d_{r}\right)^{1 / 2}, 1 \cdots, 1\right)$. Then $D^{-1} V A A^{\prime} V^{\prime} D^{-1}=D^{-1} V B B^{\prime} V^{\prime} D^{-1}$, $=\operatorname{diag}\left(I_{r}, 0_{a-r}\right)$. Write $D^{-1} V A=X$ and $D^{-1} V B=Y$, so $X X^{\prime}=Y Y^{\prime}$

${ }^{1} \mathrm{H}$. Hotelling, Relations between two sets of variates, Biometrika vol. 28 (1936) p. 321. The present paper is the basis for an extension to more than two sets. 
$=\operatorname{diag}\left(I_{r}, 0_{a-r}\right)$. This implies that $X$ has $r$ nonzero orthonormal rows. Now to the $r$ nonzero rows add $b-r$ rows chosen so as to present a $b$-rowed orthogonal matrix $X_{0}$. Do the same with $Y$ and call the resulting orthogonal matrix $Y_{0}$. Letting $U=Y_{0}^{\prime} X_{0}$, we have $X_{0}=Y_{0} U$. Hence $X=Y U$ and $A=B U$.

Let

$$
\left(\begin{array}{ll}
I_{p} & A \\
A^{\prime} & I_{q}
\end{array}\right)=\left(\begin{array}{l}
X \\
Y
\end{array}\right)
$$

be the semi-canonical form (1) of a positive definite matrix partitioned according to $n=p+q$. Let $K$ be an orthogonal matrix such that $K X X^{\prime} K^{\prime}=D$, where $D$ is diagonal. Hence $K A A^{\prime} K^{\prime}=D-I_{p}$ $=B B^{\prime}$, where $B$ is a $p$ by $q$ matrix whose first $p$ diagonal coefficients are the square roots of those of $D-I_{p}$. By the lemma there exists an orthogonal matrix $L^{\prime}$ such that $B=K A L^{\prime}$. Then the canonical form desired is obtained by using the internal transformation

$$
\left(\begin{array}{ll}
K & 0 \\
0 & L
\end{array}\right)
$$

5. Applications. These canonical forms have been applied to the determination of minimal principal minors of partitioned correlation matrices in an unpublished paper by R. G. D. Steel and the author. These minors have statistical significance as generalizations of the minors $1-b_{i}^{2}$ formed from the matrix of $\$ 4$, with $b_{i}$ a diagonal coefficient of $B$ (see footnote 1 ).

One easily proved property of the semi-canonical reduction of $\$ 1$ is the following: Among all $S P S^{\prime}$ with unit diagonal coefficients and fixed $^{2} P$, the determinant $\left|S P S^{\prime}\right|$ is maximum when $S P S^{\prime}$ is in the semi-canonical form. For, let $P_{1}$ be the first diagonal block of $P$ and let $S P S^{\prime}$ be in semi-canonical form. Then $S_{1} P_{1} S_{1}^{\prime}=I_{p}$, or $\left|S_{1}\right|^{2}$ $=1 /\left|P_{1}\right| \geqq 1$. Similarly $\left|S_{2}\right|^{2} \geqq 1,\left|S_{3}\right|^{2} \geqq 1$. Hence $|S|^{2} \geqq 1$, implying $\left|S P S^{\prime}\right| \geqq P$.

Iowa State College of Agriculture and Mechanic Arts

2 Here $P$ is a correlation matrix. 